\title{
The multivariate multiple-membership random-effect model: An introduction and evaluation
}

\author{
Sunyoung Park ${ }^{1} \cdot$ S. Natasha Beretvas ${ }^{2}$
}

Published online: 17 December 2019

(C) The Psychonomic Society, Inc. 2019

\begin{abstract}
In the present study, we focused on models that handle several data structure complexities simultaneously. We introduced and evaluated the multivariate multiple-membership random-effect model (MV-MMREM) for handling multiple-membership data in scenarios with multiple, related outcomes. Although a recent study introduced the idea of the MV-MMREM, no research has directly assessed its estimation nor demonstrated its use with real data. Therefore, we used real multiple-membership datasets that included multiple, related outcomes to demonstrate interpretation of the MV-MMREM parameters. In addition, a simulation study was conducted to assess estimation of the MV-MMREM under a number of design conditions. Also, the robustness of the results was assessed for multivariate multiple-membership data when they were analyzed using a multivariate hierarchical linear model that ignores the multiple-membership structure (MV-HLM), as well as when using multiple univariate MMREMs. The results showed that the MV-MMREM works well in comparison with both MV-HLM and univariate MMREMs when the data structure had missingness outcomes, multivariate outcomes, and multiple membership clusters. Finally, we discuss limitations of the MV-MMREM and areas for future research.
\end{abstract}

Keywords Multivariate Multiple-membership Random Effects Model (MV-MMREM) · Missing Data $\cdot$ Simulation Study $\cdot$ The Early Childhood Longitudinal Study · Kindergarten Class of 1998-99 (ECLS-K)

In any statistical analysis, it is critical to match the model being estimated with the characteristics of the data's structure. For example, if data are collected from individuals nested within clusters, such as students nested in elementary schools, then the statistical model needs to address the resulting dependency either by correcting the standard errors or by directly modeling the clustering using the multilevel model (Hox, 2010; Raundenbush \& Bryk, 2002). If individuals are members of more than one cluster, then the multiple-membership data structure should be appropriately modeled (Chung \& Beretvas, 2012; Wolff Smith \& Beretvas, 2017). Another kind of dependence that might also need to be addressed is that of multivariate outcomes. For an analysis of multiple related outcomes, power and Type I error control can be enhanced and more sophisticated research questions can be tested by using a

Sunyoung Park

sunyoungpark@callutheran.edu

1 Graduate School of Pscyhology, California Lutheran University, Thousand Oaks, CA, USA

2 Quantitative Methods Program, Educational Psychology Department, University of Texas, Austin, TX, USA multivariate rather than multiple univariate models (Snijders \& Bosker, 2012).

Typically, in empirical data especially for large-sample longitudinal data, the data have several data structure complexities at the same time. For example, the Early Childhood Longitudinal Study, Kindergarten Class of 1998-99 (ECLSK) dataset consists of students' scores on multiple achievement outcomes (e.g., reading, math, and science scores) as well as at multiple time-points and thus includes multiple possible school identifiers (e.g., first-, third-, and fifth-grade schools). Therefore, the ideal statistical model should consider not only multiple outcomes' dependency but also the possibility of multiple-membership. Analysis of this kind of dataset typically involves a sufficiently large sample size to allow estimation of more complicated statistical model. However, even though previous methodological research has focused on estimation of the multivariate multilevel model and on handling multiple-membership in data, no research has focused on estimation of a multivariate multilevel model for multiple-membership data.

Therefore, in the present study we focused on a model that handles several data structure complexities simultaneously. More specifically, this study focused on a model mentioned 
in prior research (Beretvas, 2015) that is intended to handle multiple-membership data in which individuals are members of multiple clustering units and for data that includes multiple related outcomes multilevel.

\section{Multilevel model}

Researchers can handle clustered data in several ways. In the present study, we focused on the use of the multilevel model for handling the dependency that results from data being gathered for individuals who are nested within clusters (see the more detailed treatments in Hox, 2010; Raundenbush \& Bryk, 2002). If the dependence of residuals within a cluster is ignored, then the validity of statistical inferences can be impacted largely as a function of biased standard error estimation.

Several methodological studies have focused on estimation of the conventional multilevel model. First, various studies have investigated the influence of sample size on accuracy of parameter estimation (e.g., Browne \& Draper, 2000; Clarke \& Wheaton, 2007; Kim, 1990; Maas \& Hox, 2004, 2005; McNeish \& Stapleton, 2013; Mok, 1995), the studies indicate that the number of clusters is more critical than the number of individuals within clusters for reasonable parameter and standard error recovery. Second, in terms of power (Mathieu, Aguinis, Culpepper, \& Chen, 2012; Raudenbush \& Liu, 2000; Scherbaum \& Ferreter, 2009; Snijders \& Bosker, 2012) and Type I error rates (Bassiri, 1988; Chang, 2015), at least 10 clusters with 100 subjects (per cluster), 20 clusters with 20 subjects, or 30 clusters with 30 subjects are typically recommended for two-level data for enough higher (typically, 80\%) power and nominal Type I error rate (typically, .05). Finally, the impact of balanced versus unbalanced sample size (Cools, Van den Noortgate \& Onghena, 2009; Konstantopoulos, 2010) has been investigated and authors have found that balanced sample sizes within clusters result in more stable estimates.

Use of the conventional multilevel model requires an assumption that individuals are purely nested within clusters meaning that each individual is associated with no more than one clustering unit. However, individuals are not always purely nested in single clusters. To use the conventional multilevel model, researchers have to either delete the individuals who are members of multiple clusters or ignore one of the set of clusters associated with each student. However, use of either of these ad hoc procedures reduces power and results in biased random effects variance components and standard error estimates (Chung \& Beretvas, 2012; Luo \& Kwok, 2009). One alternative approach for handling multiple-membership data is to use the multiplemembership random-effect model (MMREM; Goldstein, 2010; Rasbash \& Browne, 2001).

\section{Multiple-membership random-effect model}

The MMREM can be used to handle data in which some individuals are associated with multiple clustering units (for example, when mobile students who attend multiple schools). The unconditional MMREM is as follows, at Level 1:

$Y_{i\{j\}}=\beta_{0\{j\}}+r_{i\{j\}}$

and at Level 2, the intercept parameter is modeled as follows:

$\beta_{0\{j\}}=\gamma_{00}+\sum_{h \in\{j\}} w_{i h} u_{0 h}$

where, $\{j\}$ represents the set of Level 2 units (e.g., elementary schools) associated with each Level 1 unit (e.g., student) $i$ and $w_{i h}$ indicates the weight assigned to each Level 2 unit associated with a Level 1 unit (e.g., elementary school associated with a student). The weights should sum to one (i.e., $\sum_{h \in\{j\}} w_{i h}=1$ ) for each individual $i$ (Goldstein, 2010). For example, if a mobile student $i$ attended three elementary schools (elementary schools ES1, ES2, and ES3), the Level 2 model would be:

$$
\begin{aligned}
\beta_{0\{E S 1, E S 2, E S 3\}}= & \gamma_{00}+(1 / 3) u_{0, E S 1}+(1 / 3) u_{0, E S 2} \\
& +(1 / 3) u_{0, E S 3},
\end{aligned}
$$

where the weights of $1 / 3$ are assigned to the residual for each elementary school.

Several empirical studies have focused on use of the MMREM under various conditions. Chandola, Clarke, Wiggins, and Bartley (2005) analyzed health-related outcomes for individuals nested within households and residential districts. When they checked the deviance information criterion (DIC; Spiegelhalter, Best, Carlin, \& Van der Linde, 2002), the MMREM model had better fit than the conventional multilevel model that ignored the multiple-membership. Leckie (2009) and Goldstein, Burgess, and McConnell (2007) also reported that the variance components' estimates for upper level clustering units (schools) were underestimated when the conventional multilevel model was used and multiple-membership was ignored. Several methodological studies have also supported the results of these empirical studies. For example, Chung and Beretvas (2012) and Wolff Smith and Beretvas (2017) found that the fixed effects and Level 2 variance components were underestimated and the Level 1 variance was overestimated when the multiple-membership data structure was ignored.

The conventional multilevel model and the MMREM mentioned above focus on analyses of a single, univariate outcome. However, in practice, multiple related outcomes are typically collected and of interest to applied researchers (e.g., Reise \& Duan, 2003; Tabachnick, Fidell, \& Osterlind, 
2001). It is possible to conduct multiple univariate analyses. Unfortunately, as with simpler models and analyses, ignoring the correlations among outcomes in separate univariate analyses can introduce threats to the validity of associated statistical inferences. For example, using multiple univariate models to test each hypothesis can inflate the experimentwise Type I error rate as well as can have lower power (Baldwin, Imel, Braithwaite, \& Atkins, 2014). And if researchers are interested in not only fixed effects but also random effects variance estimation, use of a multivariate model can provide more accurate variance estimates. In addition, use of a multivariate model can result in smaller standard error estimates and thereby improve power for fixed-effect parameter estimates especially when outcomes in the multivariate model are strongly related (Snijders \& Bosker, 2012). Therefore, an extension to the univariate multilevel model, termed the multivariate multilevel model can be used that handles a set of related outcomes simultaneously.

\section{Multivariate hierarchical linear model}

The multivariate hierarchical linear model (MV-HLM) is a special case of the multilevel model that is used in scenarios with multiple related outcomes (Baldwin et al., 2014; Goldstein, 2010; Hox, 2010). As an example, a researcher might be interested in analyzing student data including scores on reading, mathematics, and science achievement. The data might be clustered such that individual students are nested in schools. For this kind of data, a three-level MV-HLM could be used with outcomes at Level 1 clustered within individuals (Level 2) who are nested within schools (Level 3). At Level 1 , the students' three outcomes (reading, mathematics, and science achievement) can be identified using dummy-coded indicator variables as follows (Hox, 2010):

$Y_{q i j}=\pi_{1 i j} d_{1 i j}+\pi_{2 i j} d_{2 i j}+\pi_{3 i j} d_{3 i j}$

where $Y_{q i j}$ is outcome $q$ score for Level 1 unit $i$ (e.g., student) within Level 2 unit $j$ (e.g., school), and $d_{q i j}$ is a dummycoded variable identifying each of three outcomes (reading, mathematics, and science achievement). In addition, $\pi_{q i j}$ is equal to $Y_{q i j}$ when the corresponding $d_{q i j}$ is equal to 1 and the other dummy coded variables equal zero. Note typically the Level 1 error is omitted from the model because level one is only used to partition the multivariate outcomes' structure in the model (Goldstein, 2010; Hox, 2010). The Level 2 model for individual students is as follows:

$\pi_{q i j}=\beta_{q 0 j}+r_{q i j}$

where the residuals $\left(r_{q i j}\right)$ are assumed to follow a multivariate normal distribution with means of zero and a variancecovariance matrix specified as follows: $\left[\begin{array}{l}r_{1 i j} \\ r_{2 i j} \\ r_{3 i j}\end{array}\right] \sim M V N\left[\left(\begin{array}{l}0 \\ 0 \\ 0\end{array}\right),\left(\begin{array}{lll}\sigma_{r .11}^{2} & \sigma_{r .12} & \sigma_{r .13} \\ \sigma_{r .21} & \sigma_{r .22}^{2} & \sigma_{r .23} \\ \sigma_{r .31} & \sigma_{r .32} & \sigma_{r .33}^{2}\end{array}\right)\right]$.

Finally, at Level 3, the model is as follows:

$\beta_{q 0 j}=\gamma_{q 00}+u_{q 0 j}$

where the residuals $\left(u_{q 0 j}\right)$ are assumed to follow a multivariate normal distribution with means of zero and a variancecovariance matrix as follows:

$\left[\begin{array}{l}u_{10 j} \\ u_{20 j} \\ u_{30 j}\end{array}\right] \sim M V N\left[\left(\begin{array}{l}0 \\ 0 \\ 0\end{array}\right),\left(\begin{array}{lll}\sigma_{u .11}^{2} & \sigma_{u .12} & \sigma_{u .13} \\ \sigma_{u .21} & \sigma_{u .22}^{2} & \sigma_{u .23} \\ \sigma_{u .31} & \sigma_{u .32} & \sigma_{u .33}^{2}\end{array}\right)\right]$

A simulation study focused on estimation of the three-level MV-HLM (Chang, 2015) was conducted to assess minimum sample sizes needed for acceptable parameter recovery. According to the results, using as few as five clusters and 30 subjects per cluster provided adequate fixed-effect parameter estimates, but more than 30 clusters and 100 subjects per cluster were required for reasonable statistical power as well as parameter recovery across conditions.

A recent simulation study focused on the performance of MV-HLM when some outcome data are missing (Chang \& Pituch, 2019). The study compared results when estimating each of the following models: (1) a MV-HLM modeling the dependencies among the multivariate outcomes, (2) a MV-HLM but assuming the multivariate outcomes are uncorrelated, and (3) multiple univariate HLMs - one for each outcome. Datasets were generated with 30 clusters and 30 individuals per cluster. The results showed that use of MV-HLM had unbiased fixedeffect estimates as well as unbiased Level 1 and Level 2 residuals' variance estimates across all conditions. However, the results also indicated that the use of the MV-HLM assuming uncorrelated outcomes and the use of multiple univariate HLMs produced bias with missing-at-random (MAR) data. The fixedeffect parameter estimates for the first outcome, which had MAR missingness, were overestimated if the correlation between outcomes was positive, whereas the fixed effects were underestimated if the correlation between outcomes was negative. The Level 1 and Level 2 residuals' variance estimates were also negatively biased under the MV-HLM with uncorrelated outcomes and multiple univariate-HLM models.

Although research has focused on the application and estimation of the three-level MV-HLM, little research has extended this conventional MV-HLM to a model that handles the mobility of some individuals across clustering units. Beretvas (2015) suggested extension of the univariate MMREM for scenarios with multivariate outcomes and multiple membership of individuals within clusters. The present study focuses on the MV-MMREM model, which merges the multivariate multilevel model (MV-HLM) and the MMREM. 


\section{Multivariate multiple-membership random effects model}

As an example of the formulation of the MV-MMREM for a scenario with three related outcomes, the Level 1 model becomes

$Y_{q i\{j\}}=\pi_{1 i\{j\}} d_{1 i\{j\}}+\pi_{2 i\{j\}} d_{2 i\{j\}}+\pi_{3 i\{j\}} d_{3 i\{j\}}$.

The Level 2 model is

$\pi_{q i\{j\}}=\beta_{q 0\{j\}}+r_{q i\{j\}}$

where the residuals $\left(r_{q i\{j\}}\right)$ are assumed to follow a multivariate normal distribution with means of zero and a variancecovariance matrix as for Eq. 5. At Level 3, to handle the multiple-membership structure, we add in weighted composites of the residuals to represent variability in each outcome, $q$, across clustering unit (e.g., elementary school) as follows:

$\beta_{q 0\{j\}}=\gamma_{q 00}+\sum_{h \in\{j\}} w_{i h} u_{0 h}$

where the set of three outcomes' Level 3 residuals are assumed to follow a multivariate normal distribution with means of zero and a variance-covariance matrix as for Eq. 6 .

\section{Missingness in MV-HLM, univariate MMREM, and MV-MMREM}

Estimation of the multivariate model can handle missing at random (and missing completely at random data) with both maximum likelihood (e.g., Chang \& Pituch, 2019) and MCMC estimation (Browne, 2017). The multivariate models, MV-HLM, and MV-MMREM can handle missing data for multiple-outcomes simultaneously whereas the univariate model, Uni-MMREM, cannot handle the missing data because the analyses are conducted separately for each outcome and information about missing outcomes from related, complete outcome data is unavailable.

Thus, it is expected that use of the Uni-MMREM will result in more biased fixed-effect estimates for MAR data than for complete data analyses due to the missingness as reported in the Chang and Pituch (2019). In addition, the Uni-MMREM estimates of residuals' variance components will be different than those estimated using the MVMMREM model for the MAR dataset. Whereas estimation of the multivariate models (MV-HLM and MV-MMREM) handles the missing data using information from the correlated outcomes, estimation of the Uni-MMREMs cannot use information from the other outcomes, because each outcome is analyzed separately. Therefore, we expect that when estimating the Uni-MMREM, the missing data will result in biased residuals' variance estimates.
Although a couple of studies have compared MV-HLM and univariate HLM, no research has directly assessed estimation of the MV-MMREM nor demonstrated its use with real data. Therefore, to demonstrate the use of MV-MMREM with real data, we used a real multiple-membership dataset that included multiple related outcomes to demonstrate interpretation of MV-MMREM parameters. In addition, we conducted a simulation study that assessed estimation of the MVMMREM and assessed robustness of results for MVMMREM datasets when analyzed using the MV-HLM and the multiple univariate-MMREMs under a number of design conditions. We hypothesized that use of the MV-MMREM should provide more accurate variance component estimates and standard error estimates across all of conditions than when using multiple univariate MMREMs or using MV-HLM. However, with the real-data analysis, we cannot infer which model's results are more accurate and instead can only identify differences. The simulation study that we also conducted will help identify which model's results are more accurate.

\section{Real-data study}

\section{Method}

Dataset The Early Childhood Longitudinal Study, Kindergarten Class of 1998-99 (ECLS-K) dataset was used that includes various types of information collected about children from kindergarten through middle school (eighth grade). Data that are gathered include children's achievement, psychological status, as well as contextual factors describing children's families, schools, teachers, and communities.

The present study focused on the students who had school identifiers for first, third, and fifth grades. For the timeframe of interest in this dataset, the maximum number of schools that students attended was three and equal weights were assigned to each school attended by mobile students. Therefore, if students attended different schools for all three time points (first, third, fifth grade), the estimation model matches that in Eq. 3. The original dataset included data for 21,409 students, however, 9,842 students (46\%) were excluded because their school identification was missing. School level identifiers cannot be missing. Thus, the full dataset included 11,567 students. The majority of these students had all three scores' values (11,030 students), although for 24 students only one or two outcome scores were available and for 507 students none of the outcome scores were available. We constructed a subset dataset containing values only for students with no missing math, reading, and science scores in fifth grade. This complete dataset $(N=10,366)$ with no missing outcome scores thus was reduce by $21 \%$ due to missing outcome scores. We then created a subset of that reference (complete) dataset into which we introduced missingness for which we 
knew the actual missing-data mechanism. In total, the data for 10,366 students from 1,856 schools were included in the final analysis. Of these students, 1,192 students (11.5\%) attended more than one school during three time points. Among these 1,192 students, only $5 \%$ of the mobile students (60 students) attended three schools. For students who attended two schools, weights of $1 / 2$ are assigned to the residual for each school and $1 / 3$ are assigned to the residual for each school for students who attended three schools. The corrected item response theory scale math, reading, and science scores were used as the outcomes. All scores on these measures have been previously reported as having relatively high reliability, exceeding .8 (Pollack, Atkins-Burnett, Najarian, \& Rock, 2005).

Estimating models Differences in estimates as a function of model used were assessed by estimating the following three models using the same dataset: (1) three-level MV-HLM ignoring multiple-membership (only use fifth-grade school ID), (2) multiple two-level Uni-MMREMs ignoring multiple outcomes' dependency, and (3) three-level MV-MMREM. All models were estimated using the MLwiN software (Rasbash et al., 2000) with Markov chain Monte Carlo (MCMC) estimation. Three chains were run with 50,000 iterations with a burn-in of 10,000 .

The fixed effects' and random effects' variance components and $S E$ estimates, as well as the per-outcome intraclass correlation coefficient (ICC) estimates, were compared across the three models. Also, the DIC (Spiegelhalter et al., 2002) values were used to compare the fits of the different models investigated. Smaller DIC values support better model fit. However, note that because the DIC is a function of $-2 * \log$ likelihood values, smaller datasets (here, the MAR dataset) lead to larger $-2 * \log$ likelihood values and lower DIC values, regardless of the model fit. Thus, we only compared the DIC for MV-HLM versus MV-MMREM models estimated within the dataset. MV-MMREM and MV-HLM estimates of the Level 1 and Level 2 correlations among residuals for pairs of outcomes were estimated and compared. These correlations are not estimated with the univariate MMREMs.

Missing-at-random data As we mentioned above, one of the main advantages of a multivariate versus a univariate analysis is that the multivariate approach can handle missing outcome data. Therefore, one of the conditions that we considered in the simulation study was the proportion of missingness on the outcomes. To align the real data analysis with the simulation study, we also compared these three models' estimates using the dataset without missing data and again after we introduced missing-at-random (MAR) data into two of the three outcomes demonstrate the expected impact of not using the MV-MMREM when there are MAR data. Future research can explore model estimation in scenarios with other types of missing data (e.g., missing-not-at-random data).
For the MAR dataset, $20 \%$ of math and $20 \%$ of science scores were deleted with lower reading scores leading to more likely MAR math and science scores. The missing outcome data were obtained by following the same processes as used in previous research (Chang \& Pituch, 2019; Enders, 2001). For each student, the probability of each math and science score being missing was calculated as one minus the percentile rank for the individual's (nonmissing) reading score. Then, a random number sampled from a $[0,1]$ uniform distribution was generated for each student's math and their science scores. Each random number was then compared to the corresponding probability of missingness (one minus the percentile rank). If the probability of missingness was larger than the random number then the relevant outcome score was removed to create the MAR dataset but not more than $20 \%$ of math and science scores were deleted.

\section{Results}

Fixed-effect parameters, standard error estimates, and variance components As a reminder, three datasets were analyzed including the full dataset, the complete dataset (referring to its inclusion of students for whom all three outcomes were reported) and the MAR dataset that was a subset of the complete dataset into which MAR was introduced into the outcomes. For the full and complete datasets, fixed-effect parameter estimates did not differ substantially across the three models estimated (see Table 1). For the analyses of the MAR dataset, however, fixed-effect parameter and $S E$ estimates were impacted when estimating multiple univariate-MMREMs. The estimates of univariate-MMREMs had about $6 \%$ larger fixed effects and smaller (ranged from $2 \%$ for reading score to $20 \%$ for science score) $S E$ estimates than were found when using MV-MMREM for estimation.

For the full and complete datasets, the multiple univariateMMREM estimates of school- and student-level random effects variance components were similar in value to their corresponding MV-MMREM estimates. However, the variance components from the analysis of the full dataset were about $2 \%$ and $10 \%$ larger for student- and school-level variances than the respective values from the analysis of the complete dataset. As expected (Chung \& Beretvas, 2012; Goldstein, 2010), MV-HLM's estimates of school-level random effects variance and covariance components were about $7 \%$ smaller than MV-MMREM estimates for analyses of both the full and complete datasets. Also, the analyses of the MAR-dataset showed substantially smaller Level 2 (ranged from $0 \%$ for reading score to $24 \%$ smaller for science score) and Level 3 (about $8 \%$ across the three outcomes) variance components than MV-MMREM estimates for the math and science outcomes (which had missing data) (see Table 1).

For models estimated using the full dataset, the MVMMREM had a smaller DIC value $($ DIC $=288,047)$ than 


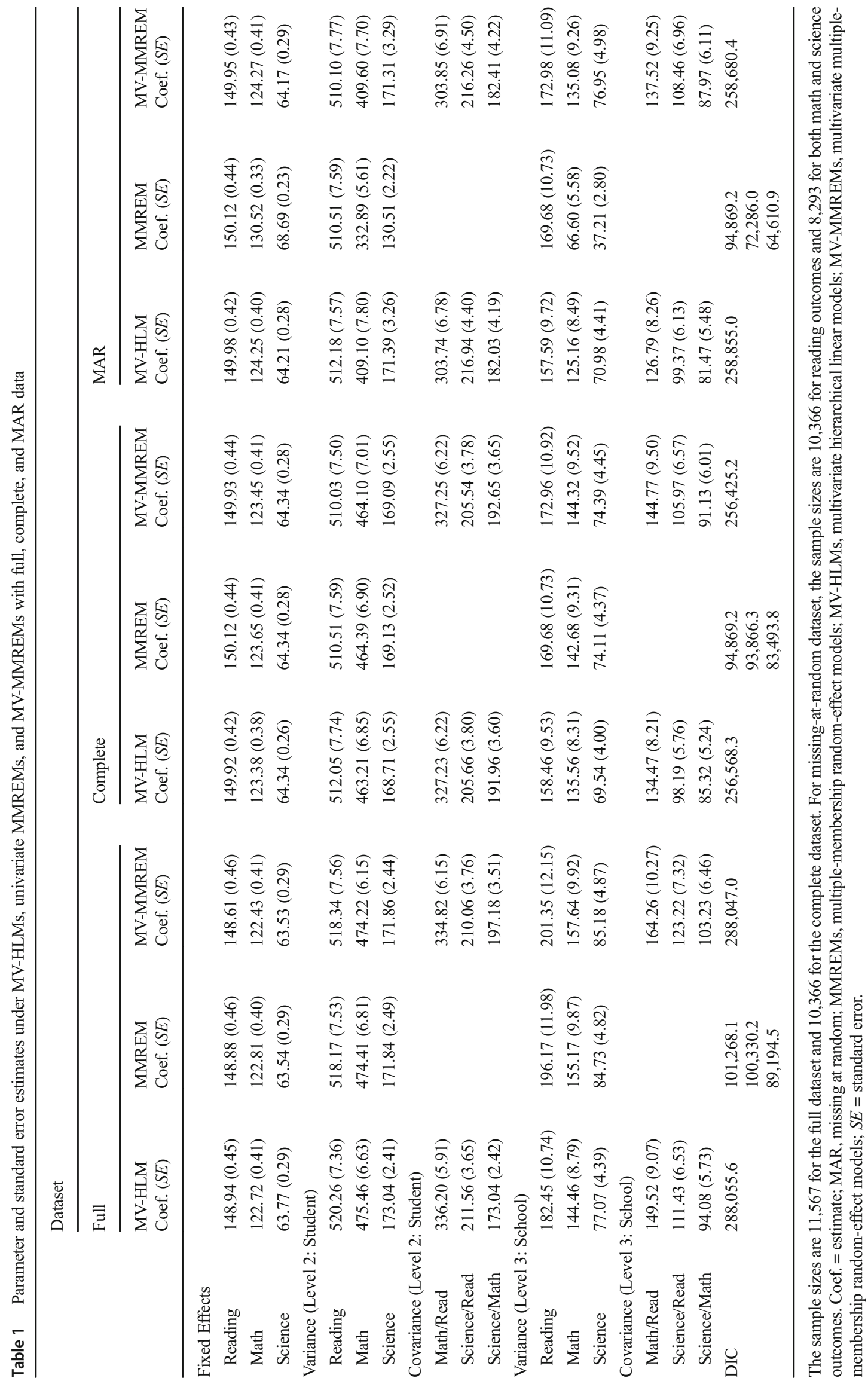


did the MV-HLM (DIC = 288,055), supporting the better fit of the MV-MMREM. In addition, for models estimated using the complete dataset, the MV-MMREM had a much smaller DIC value $($ DIC $=256,425)$ than for the MV-HLM (DIC = 256,568) supporting the better fit of the MV-MMREM. As we mentioned above, the DIC values for univariate MMREM were not used to compare model fit. The same pattern supporting the better fit of the MV-MMREM (DIC = 258,680 ) over the MV-HLM (DIC $=258,855$ ) was found for the MAR dataset (see Table 1).

Correlation among outcomes' residuals and ICCs The correlations among outcomes' residuals at the school level were larger in magnitude than the corresponding student level correlations. Note that the correlations among outcomes for all three models were the same to two decimal places for the MV-HLM and MV-MMREM estimates. As expected, the correlations among academic achievement score residuals for reading, math, and science were moderate to high in value. The correlations ranged from .67 to .70 for student level and from .88 to .93 for school level residuals. Next, the ICCs were calculated for each outcome for the three estimating models. The science score had the largest ICC (.29-.31), ICCs for Reading score ranged from .24 to .25 and the math score had the smallest ICC (.23-.24) across the three models.

In summary, for analyses of all three datasets, school-level variance components under MV-HLM or multiple univariate MMREMs were smaller than variance components estimated using the MV-MMREM. For analyses of the MAR dataset, estimation of multiple univariate MMREMs resulted in larger fixed-effect and smaller variance component parameter estimates for math and science scores when compared with the other two models' estimates. However, even though the full and complete datasets provided one reference point to compare for estimates with MAR dataset analyses, the full and complete dataset results were still based on an analysis of a real dataset for which we do not know the true parameters nor true mechanism for missing data. Also, because the real data analysis was conducted using a relatively larger sample size, it is still unknown what kind of sample sizes are necessary for reasonable parameter recovery for the MV-MMREM. Therefore, we also conducted a simulation study to examine parameter recovery under the MV-MMREM and to assess robustness of estimates when misspecifying the MVMMREM data's structure and model.

\section{Simulation study}

\section{Method}

As a first study focused on evaluating estimation of the unconditional MV-MMREM, the present study generated datasets to fit the MV-MMREM. In this study, we examined differences in results when handling multiple-membership clusters, multivariate outcomes data using (1) a conventional three-level MV-HLM recognizing only the most recent school attended (fifth grade) and ignoring the multiple-membership data structure (see Eqs. 4, 5, and 6), (2) multiple two-level univariate MMREMs - one for each of the multivariate outcomes (see Eqs. 1 and 2), and (3) a three-level MV-MMREM (see Eqs. 7, 8, and 9).

\section{Generating parameters and conditions}

When generating data, the values for the true mean for each of three outcomes ( $\gamma_{q 00}$ in Eq. 9) of 150, 100, and 50, respectively, were used, which provide values close to those estimated using the real data analysis conducted in this study. The Level 2 (student-level) variance $\left(\sigma_{r}^{2}\right)$ values were also generated using values close to those found in the real data analyses in this study including values of 500, 450 , and 150 , respectively. In the simulation study we manipulated seven conditions, including the number of schools $(k)$, the number of students per school $(n)$, the percentage of students who changed schools (mobility rate), the correlation among outcomes' residuals, ICC, type of missingness, and proportion of missing outcomes.

Number of schools (k) In previous studies focused on the MMREM (Chung \& Beretvas, 2012; Wolff Smith \& Beretvas, 2014) and in a study focused on estimation of the multivariate multilevel model (Chang, 2015), authors recommended that the minimum number of higher level clustering units be at least 50 units. Therefore, the present study investigated scenarios with 50 and 100 clusters (schools).

Number of students per schools ( $\mathrm{n}$ ) Previous, related methodological studies have investigated scenarios with 20 and 40 students per cluster (Meyers \& Beretvas, 2006; Shi, Leite, \& Algina, 2010; Wolff Smith \& Beretvas, 2014). In keeping with this previous research, the present study used the same values for the number of level one units (students) per level two unit (school). Also, the number of students per school within a real dataset typically vary, thus, we sampled the number of students per school from a uniform distribution for each condition. We used two values for this condition sampling from uniform distributions with means of 20 and 40 students per school and ranging, respectively, from 10 to 30 and 30 to 50 .

Mobility rate In the present study we explored a scenario in which the clustering units across three-time points are known for the individuals (students). Thus, the maximum number of schools attended for a mobile student would be three. In the ECLS-K data, about $10 \%$ of students changed schools during 
three time points. In addition, previous studies have investigated $10 \%$ and $20 \%$ as small to moderate degrees of student mobility (Chung \& Beretvas, 2012; Wolff Smith \& Beretvas, 2014). Therefore, in line with these previous simulation studies and with the real data, conditions with $10 \%$ as the small and $40 \%$ as high mobility rates were explored. Inclusion of conditions with the high mobility rate, the simulation study allowed us to assess the robustness of MV-HLM and univariate MMREMs in scenarios with more extreme discrepancies between models and the data's actual structure. Among the students who change the schools $(10 \%$ or $40 \%)$, some of the mobile students attend two different schools while others attend three different schools across three time points.

In terms of proportion of students who attend three different schools across three time-points, the actual ECLS-K data examined here indicated that only $5 \%$ of mobile students attended three different schools. However, in previous applied research (e.g., Gruman, Harachi, Abbott, Catalano, \& Fleming, 2008; Kerbow, 1996) and in related methodological work (Wolff Smith \& Beretvas, 2014), there was evidence of a higher percentage $(40 \%)$ of mobile students attending different schools at different time points. Therefore, in the present study we also examined conditions with the larger percent mobility value, $40 \%$ of mobile students were simulated to attend three different schools. Under conditions with $10 \%$ mobility, $90 \%, 6 \%$, and $4 \%$ were used as the percentage of students simulated to attend one, two, and three schools, respectively. Also, under conditions with $40 \%$ mobility, $60 \%$, $24 \%$, and $16 \%$ were used as the percentage of students attending one, two, and three schools. After randomly assigning mobility, the schools' identification numbers for each student were assigned on the basis of the number of schools attended. When the students were assigned to attend two schools, a value of one was added to the school identifier for third grade to obtain the fifth-grade school identifier.

\section{Correlations among outcomes' residuals at Level 2 and Level}

3 In ECLS-K data, the correlations among outcomes' residuals were found to range from .67 to .70 at Level 2 and from .88 to .93 at Level 3. As a first study of the MV-MMREM, we investigated a more limited set of generating values for the correlations among pairs of residuals. The correlations among outcomes' residuals at Level 2 and Level 3 were generated with moderate (.3 at Level 2 and .5 at Level 3 ) and large (.5 at Level 2 and .7 at Level 3) values. Also, as demonstrated in the real data analysis results, the correlation among outcomes' residuals could differ depending on the outcome. That is, with three outcomes, we used values of .2 (between Outcomes 1 and 2), .3 (between Outcomes 2 and 3), and .4 (between Outcomes 1 and 3 ) at Level 2, and .4 (between Outcomes 1 and 2), .5 (between Outcomes 2 and 3), and .6 (between Outcomes 1 and 3 ) at Level 3 in the moderate correlations condition. In the large correlations condition, we used values of .4 (between Outcome 1 and 2), .5 (between Outcome 2 and 3 , and .6 (between Outcomes 1 and 3) at Level 2, and .6 (between Outcomes 1 and 2), .7 (between Outcomes 2 and 3), and .8 (between Outcomes 1 and 3 ) at Level 3.

Intraclass correlation coefficients According to the actual data analyses results reported above, the ICC estimates ranged in value from $23 \%$ to $31 \%$. Also, ICC values of $5 \%$ and $15 \%$ were considered in Chung and Beretvas (2012) and 15\% and $25 \%$ were used in Wolff Smith and Beretvas (2014). In the simulation study focused on the three-level multivariate HLM (Chang, 2015), the author used 10\% and 30\% matching previous studies focused on the two-level HLM (Hedges \& Hedberg, 2007; Maas \& Hox, 2004, 2005). The present study used $15 \%$ and $25 \%$ as small and large values for the ICC. Because the present study investigated the MV-MMREM with three outcomes, the ICC values for each outcome might differ. Therefore, we investigated two conditions with the relatively smaller ICC values conditions (with ICC values of $10 \%, 15 \%$, and $20 \%$ for the different outcomes) and the relatively larger ICC values (ICC $=20 \%, 25 \%$, and $30 \%$ ). On the basis of the condition-specific values for the ICC and correlations, the Level 2 covariance, Level 3 variance and covariance values were derived.

Type of missingness To compare the performance of three models under conditions with missing data, the type of missingness was varied. In addition to the complete dataset without any missing data condition, two missing mechanisms were assessed including: missing-completely-at-random (MCAR) and MAR data. Note that outcome one was always complete across conditions. In the MCAR conditions, the values of Outcomes 2 and 3 were randomly selected for deletion using the condition-specific proportion of missingness. For the MAR conditions, we followed the procedure used in a previous, related study (Chang \& Pituch, 2019).

The procedure for creating the MAR data is as follows. First, complete scores for Outcomes 1, 2, and 3 were generated for each subject using Eqs. 7, 8, and 9. Second, for each student, the probability of a score being missing $(P)$ for Outcome 2 and for Outcome 3 was calculated as one minus the percentile rank for the individual's score on Outcome 1. This means that if a (simulated) individual's Outcome 1 score is low, there is a higher chance of the individual having a missing Outcome 2 and Outcome 3 score. Third, a random number $(R)$ sampled from a $[0,1]$ uniform distribution was generated for each student for Outcome 2 and again for Outcome 3. This $R$ value is then compared to the probability of missingness $(P)$ that was generated at the second step. If the probability of missingness $(P)$ is larger than the random number $(R)$ then the relevant outcome score is removed from the MAR dataset. Finally, beginning with the lowest Outcome 1 scores, a student scores on Outcome 2 and Outcome 3 were 
deleted if the missing indicator (D) is 1 , and the deletion process continued until the desired amount of missing data was obtained. Because we only generated data with positive correlations among outcomes, a simulated participant with lower scores on Outcome 1 tended to have lower scores on Outcomes 2 and 3. Consequently, the deleted scores for Outcome 2 and Outcome 3 tended to be low as they were conditioned on Outcome 1 scores.

Percentage of missing outcomes Previous methodological studies investigating multivariate HLM estimation with missing data have investigated scenarios with 10\%-30\% missingness (Chang \& Pituch 2019; Park, Pituch, Kim, Chung, \& Dodd, 2015). An empirical study (Pituch, Whittaker, \& Chang, 2016) demonstrated use of multivariate HLM models with normal and binary responses used an empirical dataset in which about $42 \%$ of subjects had at least one missing outcome. Therefore, in the present study we looked at percent-missing condition values of $20 \%$ and $40 \%$. Also, as we found in our real data analysis, the proportion of missingness could differ depending on the outcomes. Therefore, for the $20 \%$ missing condition, $10 \%$ of Outcome 2 scores were missing and $30 \%$ of Outcome 3 scores were missing. And, for the $40 \%$ missing condition, $30 \%$ of Outcome 2 and $50 \%$ of Outcome 3 were missing, respectively.

In summary, we generated data to fit the combination of parameter and design condition values for each of the 160 scenarios including 32 full dataset scenarios [Number of Level 3 Units $(2) \times$ Number of Level 2 Units $(2) \times$ Student Mobility $(2) \times$ Correlation Among Outcomes (2) $\times$ ICC (2)] and 128 missing data scenarios $[32$ Scenarios $\times$ Type of Missingness (2) $\times$ Proportion of Missingness (2)]. For each condition, we generated 1,000 datasets, resulting in a total of 160,000 datasets using R software (R Development Core Team, 2010).

The R2MLwiN macro that accesses MLwiN software using an $\mathrm{R}$ interface (Zhang, Parker, Charlton, Leckie, \& Browne, 2016) was used to estimate each of the three models for each generated dataset. Each model was estimated using MCMC estimation and noninformative priors matching MLwiN's defaults. By default, in the MLwiN program for MCMC estimation, the following priors are used: (1) uniform distribution for fixed effects parameters, (2) gamma distribution for scalar variances, and (3) the Wishart distribution for covariance matrices (Browne, 2017). The three models that were estimated include: (1) MV-MLM model, (2) multiple univariate MMREMs (one for each outcome), and (3) MVMMREM.

We ran pilot analyses entailing five replications per condition to validate use of this number of iterations and burn-in values and used three chains, 10,000 iterations, and a burn-in of 1,000. We kept track of Rhat (Gelman \& Rubin, 1992) values for the full-scale simulation study to tally the proportion of non-convergent cases. If overall Rhat values were greater than 1.1 for any condition, we generated new replications to base final results on converged solutions.

\section{Analyses}

Relative parameter bias was used as an index to quantify parameter recovery. In the present study, parameter recovery for the three outcomes' fixed-effect parameter estimates $\left(\gamma_{q 00}\right)$, the Level $2\left(\sigma_{r}^{2}\right)$ and Level $3\left(\sigma_{u}^{2}\right)$ random effects' variances, and the covariances at Level $2\left(\sigma_{r q q *}\right)$ and Level $3\left(\sigma_{u q q_{*}^{*}}\right)$ were assessed. The relative standard error bias was also calculated for the $S E$ s of each of the outcome-specific fixed-effect parameters. Hoogland and Boomsma (1998) suggested that if the parameter's relative bias exceeds $5 \%$ in magnitude and if the relative standard error bias exceeds $10 \%$ in magnitude, then substantial bias can be inferred.

\section{Results}

Convergence rate Under most of the conditions examined in this study, multivariate convergence rate (Rhat) values were smaller than 1.1 supporting close to $100 \%$ convergence rates. The smallest convergence rate was $92 \%$ in conditions with the smaller ICC value $(\mathrm{ICC}=.15)$ and fewer schools $(k=50)$. The average multivariate Rhat for each estimating model and each parameter ranged from 1.00 to 1.02 across conditions.

DIC values DIC values for MV-MMREM estimates were consistently smaller by at least ten points than DIC values for the MV-HLM across all conditions. In conditions in which there were fewer schools $(k=50)$, fewer students per school $(n=$ $20)$, lower mobility (10\%), and the smaller ICC value (ICC $=$ .15), the difference in DIC values between MV-MMREM and MV-HLM become smaller (with a median difference of 15.0) but the DIC still consistently favored the MV-MMREM over the MV-HLM.

Fixed-effect and standard error estimates Note that we reported the results only when we found substantial bias using Hoogland and Boomsma's (1998) criteria (greater than 5\% for $R P B$ and than $10 \%$ for $R S E B$ ). No substantial relative parameter bias $(R P B)$ was found in estimates of the three fixed-effect estimates under both the MV-HLM and MVMMREM ( $R P B$ values ranged from $-0.2 \%$ to $4.0 \%$ ). However, when estimating the three Uni-MMREMs, fixedeffect estimates for the third outcome were substantially positively biased $(7.1 \%$, ranging from $7.0 \%$ to $7.4 \%$ ) with MAR missingness when there was a higher percentage of missingness (40\%), and stronger correlations among the outcomes (.5 at Level 2, and .7 at Level 3) (see Table 2). As we mentioned above, under the $40 \%$ missing conditions, $50 \%$ of 
Table 2 Relative parameter bias $(R P B, \%)$ for fixed effects by condition, ${ }^{\text {a }}$ estimated using univariate multiple-membership random-effect models

\begin{tabular}{|c|c|c|c|c|c|c|c|}
\hline Correlation & ICC & School & Student & Mobile & Outcome 1 & Outcome 2 & Outcome 3 \\
\hline \multirow[t]{16}{*}{$.3 / .5$} & \multirow[t]{8}{*}{$15 \%$} & \multirow[t]{4}{*}{50} & \multirow[t]{2}{*}{20} & $10 \%$ & 0.0 & 1.8 & 4.4 \\
\hline & & & & $40 \%$ & 0.0 & 1.7 & 4.3 \\
\hline & & & \multirow[t]{2}{*}{40} & $10 \%$ & 0.0 & 1.7 & 4.3 \\
\hline & & & & $40 \%$ & 0.0 & 1.6 & 4.3 \\
\hline & & \multirow[t]{4}{*}{100} & \multirow[t]{2}{*}{20} & $10 \%$ & 0.0 & 1.7 & 4.4 \\
\hline & & & & $40 \%$ & 0.0 & 1.7 & 4.4 \\
\hline & & & \multirow[t]{2}{*}{40} & $10 \%$ & 0.0 & 1.7 & 4.2 \\
\hline & & & & $40 \%$ & 0.0 & 1.6 & 4.2 \\
\hline & \multirow[t]{8}{*}{$25 \%$} & \multirow[t]{4}{*}{50} & \multirow[t]{2}{*}{20} & $10 \%$ & 0.0 & 1.8 & 4.4 \\
\hline & & & & $40 \%$ & 0.0 & 1.7 & 4.5 \\
\hline & & & \multirow[t]{2}{*}{40} & $10 \%$ & 0.0 & 1.7 & 4.3 \\
\hline & & & & $40 \%$ & -0.1 & 1.6 & 4.1 \\
\hline & & \multirow[t]{4}{*}{100} & \multirow[t]{2}{*}{20} & $10 \%$ & 0.0 & 1.8 & 4.5 \\
\hline & & & & $40 \%$ & 0.0 & 1.7 & 4.5 \\
\hline & & & \multirow[t]{2}{*}{40} & $10 \%$ & -0.1 & 1.6 & 4.2 \\
\hline & & & & $40 \%$ & 0.0 & 1.6 & 4.2 \\
\hline \multirow[t]{16}{*}{$.5 / .7$} & \multirow[t]{8}{*}{$15 \%$} & \multirow[t]{4}{*}{50} & \multirow[t]{2}{*}{20} & $10 \%$ & 0.0 & 3.4 & 7.2 \\
\hline & & & & $40 \%$ & 0.0 & 3.3 & 7.1 \\
\hline & & & \multirow[t]{2}{*}{40} & $10 \%$ & 0.0 & 3.3 & 7.0 \\
\hline & & & & $40 \%$ & 0.0 & 3.3 & 7.0 \\
\hline & & \multirow[t]{4}{*}{100} & \multirow[t]{2}{*}{20} & $10 \%$ & 0.0 & 3.3 & 7.2 \\
\hline & & & & $40 \%$ & 0.0 & 3.4 & 7.2 \\
\hline & & & \multirow[t]{2}{*}{40} & $10 \%$ & 0.0 & 3.3 & 7.1 \\
\hline & & & & $40 \%$ & 0.0 & 3.3 & 7.1 \\
\hline & \multirow[t]{8}{*}{$25 \%$} & \multirow[t]{4}{*}{50} & \multirow[t]{2}{*}{20} & $10 \%$ & 0.0 & 3.4 & 7.4 \\
\hline & & & & $40 \%$ & -0.1 & 3.3 & 7.2 \\
\hline & & & \multirow[t]{2}{*}{40} & $10 \%$ & 0.0 & 3.2 & 7.0 \\
\hline & & & & $40 \%$ & 0.0 & 3.3 & 7.0 \\
\hline & & \multirow[t]{4}{*}{100} & \multirow[t]{2}{*}{20} & $10 \%$ & 0.0 & 3.4 & 7.4 \\
\hline & & & & $40 \%$ & 0.0 & 3.4 & 7.2 \\
\hline & & & 40 & $10 \%$ & 0.0 & 3.3 & 7.1 \\
\hline & & & & $40 \%$ & 0.0 & 3.2 & 7.0 \\
\hline
\end{tabular}

The $R P B$ values are bolded when the values are larger than $5 \%$ in magnitude. ${ }^{\text {a }}$ Results are summarized only for MAR missingness conditions because the other missingness conditions had no substantial bias. Results are also only presented for the $40 \%$ missingess conditions, because there is no substantial bias for the $20 \%$ missingness conditions.

Outcome 3 scores were deleted, whereas $30 \%$ of Outcome 2 values were deleted resulting in substantial bias only for Outcome 3 estimates. The positive bias indicated that the estimates of intercept for Outcome 3 were substantially larger than the true estimates of the Outcome 3 scores.

In terms of the relative standard error bias $(R S E B)$ for the three fixed-effect parameter estimates, most of the UniMMREMs and MV-MMREM RSEB values did not exceed $10 \%$ in magnitude. However, under the MV-HLM the standard errors estimated for the second and third outcomes' fixed effects were substantially negatively biased in conditions with more mobility ( $-11.9 \%$ for Outcome 2 , and $-12.8 \%$ for Outcome 3 ) as compared with conditions with less mobility
(- 2.0\% for Outcome 2, and $-2.0 \%$ for Outcome 3 ). The full results can be obtained from the first author.

Variance and covariance component estimates MV-HLM and MV-MMREM estimates of Level 2 variances were not substantially biased. However, when estimating the three UniMMREMs Level 2 variances for Outcomes 2 and $3\left(\sigma_{r .22}^{2}\right.$ and $\sigma_{r .33}^{2}$, respectively) particularly for conditions with MAR missingness and when correlations among outcomes were stronger, the Level 2 variance estimates were underestimated. With MAR data, the $R P B$ for the Level 2 variance for Outcome 2 residuals $(-5.6 \%)$ and for Outcome $3(-9.0 \%)$ 
were negatively biased when the Level 2 residuals' correlations among pairs of outcomes were stronger $(\rho=.5)$. When the Level 2 correlation was weaker $(\rho=.3)$, bias in MAR conditions was not substantial $(R P B=-1.2 \%$ for Outcome 2, $R P B=-3.1 \%$ for Outcome 3 ).

The MV-MMREM resulted in no substantial bias in Level 3 variance estimates. Level 3 covariance estimates were positively biased in conditions with a smaller number of schools with $R P B$ values up to $9.6 \%$. Under the MV-MMREM, the mean $R P B$ for Level 3 covariance estimates in the smaller number of schools conditions ranged from $4.1 \%$ up to $9.6 \%$ whereas with the larger number schools conditions the $R P B$ was $0.6 \%$ up to $5.5 \%$. However, MV-HLM estimates of the Level 3 variances and covariances were substantially negatively biased across all conditions (see Table 3 for variances and Table 4 for covariances). The negative bias was much worse in conditions with a higher proportion of mobile students (-30.2\% for variances, $-30.1 \%$ for covariances) versus a smaller proportion of mobile students (- $7.2 \%$ for variances, $6.4 \%$ for covariances). When 100 schools were included in the datasets, this bias was slightly worse (-20.0\% for variances, $19.7 \%$ for covariances) than in scenarios in which 50 schools were included in the datasets (- $17.4 \%$ for variances, $-16.8 \%$ for covariances), resulting in about $2.5 \%$ differences between the two conditions. As can be seen in Table 5, Uni-MMREMs also resulted in negatively biased variance estimates for Outcomes 2 and 3 for MAR data (- $9.9 \%$ and - 13.2\%, respectively). With MAR datasets, the bias was worse in conditions when the true correlation among outcomes was larger ($16.3 \%)$ versus smaller $(-6.8 \%)$ across outcomes two and three.

Table 6 provides estimation results for the three models. As can be seen in the table, MAR (rather than MCAR) missingness, a higher proportion of missingness, and stronger correlations among outcomes were substantial sources of biases when using the uni-MMREM for multivariate outcomes and multiple-membership data. For the MV-HLM, we found substantial negative bias (underestimation) across all conditions in the Level 3 variance and covariance estimates. Finally, when using the MV-MMREM, most estimates were not substantially biased. However, some Level 3 covariance estimates were substantially biased in scenarios with fewer schools.

\section{Discussion}

In the present study we investigated the use and estimation of the multivariate multiple-membership random-effect model to handle multiple related outcomes and multiple-membership clusters simultaneously. This study also illustrated and assessed the impact of failing to handle both the multivariate outcomes and the multiple-membership data structure. We compared results when the multiple-membership structure is ignored and when the multiple-membership structure is recognized but the multivariate outcomes are ignored. Three models' estimates were compared using a dataset without missingness and again after some of the scores for two of the three outcomes were selected to be missing at random. We first demonstrated estimation of the three models with a real dataset. We also conducted a simulation study that evaluated the performance of three models under various conditions.

Because the pattern of results for the real dataset matched those in the simulation study, we provide a fuller explanation using results from the simulation study. In the simulation study, the convergence rate results under MCMC estimation across the three models were not problematic under most conditions. However, in conditions with smaller true ICC values and fewer schools, the convergence rates were slightly lower and the lowest convergence rate was $92 \%$. Even though a previous study (Chang \& Pituch, 2019) used maximum likelihood estimation in a simulation study that compared use and estimation of a multivariate HLM versus multiple univariate HLMs, the convergence results were similar in that the smallest convergence rates were found in conditions with smaller ICC values and more missing data. That is, estimation is challenging regardless of estimation methods with smaller ICC values, more missing data and smaller number of upper level units (Beretvas \& Murphy, 2013; Chang \& Pituch, 2019; Hox \& Maas, 2001; Whittaker \& Furlow, 2009). In addition, the low convergence rates for the MCMC estimates could have resulted from an insufficient number of iterations being used even though the multivariate Rhat values were smaller than 1.1. Therefore, for data with smaller ICC values, and fewer upper level clustering units, use of a higher number of iterations for MCMC estimation might improve convergence rates. As an additional simulation, we checked convergence rates when using a larger number of iterations (three chains, 20,000 iterations, and a burn-in of 2,000) for the condition that had the lowest convergence rate $(92 \%)$. The results indicated that the convergence rates then became $100 \%$ when using a larger number of iterations. Thus, applied researchers are encouraged to use a larger number of iterations to help improves convergence rates.

In terms of model fit, assessed by comparing DIC values, results consistently supported better fit using the correctly specified MV-MMREM over the MV-HLM across conditions. These DIC results matched results from a previous study (Chung \& Beretvas, 2012) that found that DIC values for the MMREM model estimated were consistently lower than the DIC values for conventional HLMs estimated using datasets with a multiple-membership data structure.

In terms of fixed-effect parameters, no substantial relative parameter bias was found in estimates of the three fixed effects under both the MV-HLM and the MV-MMREM. Substantial 
Table 3 Relative parameter bias $(R P B, \%)$ for Level 3 variances by condition, ${ }^{\text {a }}$ estimated using the multivariate hierarchical linear models

\begin{tabular}{|c|c|c|c|c|c|c|c|}
\hline Correlation & ICC & School & Student & Mobile & $\sigma_{u .11}^{2}$ & $\sigma_{u .22}^{2}$ & $\sigma_{u .33}^{2}$ \\
\hline \multirow[t]{16}{*}{$.3 / .5$} & \multirow[t]{8}{*}{$15 \%$} & \multirow[t]{4}{*}{50} & \multirow[t]{2}{*}{20} & $10 \%$ & -3.9 & -5.2 & -5.1 \\
\hline & & & & $40 \%$ & -26.7 & -27.7 & -28.1 \\
\hline & & & \multirow[t]{2}{*}{40} & $10 \%$ & -5.4 & -5.4 & -4.1 \\
\hline & & & & $40 \%$ & -29.3 & -28.5 & -28.8 \\
\hline & & \multirow[t]{4}{*}{100} & \multirow[t]{2}{*}{20} & $10 \%$ & -8.5 & -10.0 & -8.9 \\
\hline & & & & $40 \%$ & -31.9 & -31.9 & -31.0 \\
\hline & & & \multirow[t]{2}{*}{40} & $10 \%$ & -7.1 & -8.0 & -7.7 \\
\hline & & & & $40 \%$ & -31.0 & -32.6 & -31.2 \\
\hline & \multirow[t]{8}{*}{$25 \%$} & \multirow[t]{4}{*}{50} & \multirow[t]{2}{*}{20} & $10 \%$ & -3.0 & -4.9 & -6.8 \\
\hline & & & & $40 \%$ & -27.9 & -29.8 & -30.3 \\
\hline & & & \multirow[t]{2}{*}{40} & $10 \%$ & -4.6 & -4.7 & -6.6 \\
\hline & & & & $40 \%$ & -27.9 & -29.6 & -30.2 \\
\hline & & \multirow[t]{4}{*}{100} & \multirow[t]{2}{*}{20} & $10 \%$ & -7.5 & -8.3 & -9.1 \\
\hline & & & & $40 \%$ & -30.8 & -32.2 & -32.3 \\
\hline & & & \multirow[t]{2}{*}{40} & $10 \%$ & -7.0 & -8.8 & -9.2 \\
\hline & & & & $40 \%$ & -30.7 & -32.5 & -32.4 \\
\hline \multirow[t]{16}{*}{$.5 / .7$} & \multirow[t]{8}{*}{$15 \%$} & \multirow[t]{4}{*}{50} & \multirow[t]{2}{*}{20} & $10 \%$ & -3.5 & -4.7 & -4.8 \\
\hline & & & & $40 \%$ & -25.8 & -26.8 & -27.7 \\
\hline & & & \multirow[t]{2}{*}{40} & $10 \%$ & -6.3 & -7.2 & -5.8 \\
\hline & & & & $40 \%$ & -29.2 & -29.6 & -29.0 \\
\hline & & \multirow[t]{4}{*}{100} & \multirow[t]{2}{*}{20} & $10 \%$ & -8.9 & -9.6 & -8.2 \\
\hline & & & & $40 \%$ & -31.3 & -32.1 & -31.4 \\
\hline & & & \multirow[t]{2}{*}{40} & $10 \%$ & -9.2 & -8.6 & -7.8 \\
\hline & & & & $40 \%$ & -31.4 & -32.0 & -31.4 \\
\hline & \multirow[t]{8}{*}{$25 \%$} & \multirow[t]{4}{*}{50} & \multirow[t]{2}{*}{20} & $10 \%$ & -3.4 & -5.6 & -6.8 \\
\hline & & & & $40 \%$ & -28.6 & -29.4 & -29.9 \\
\hline & & & \multirow[t]{2}{*}{40} & $10 \%$ & -2.8 & -5.1 & -5.2 \\
\hline & & & & $40 \%$ & -28.5 & -29.8 & -30.4 \\
\hline & & \multirow[t]{4}{*}{100} & \multirow[t]{2}{*}{20} & $10 \%$ & -7.0 & -9.4 & -9.5 \\
\hline & & & & $40 \%$ & -30.7 & -31.7 & -32.4 \\
\hline & & & 40 & $10 \%$ & -7.4 & -8.3 & -9.9 \\
\hline & & & & $40 \%$ & -30.0 & -31.8 & -32.2 \\
\hline
\end{tabular}

The $R P B$ values are bolded when the values are larger than $5 \%$ in magnitude. ${ }^{\text {a }}$ Results are summarized only for MAR missingness conditions because the other missingness conditions (MCAR or Full dataset) had similar patterns. Results are presented only for the $40 \%$ missingess conditions; the pattern of results corresponded to the results with $20 \%$ missingness, although the bias was worse with $40 \%$ missingness.

positive bias was found for the Uni-MMREM estimates in scenarios with a higher proportion of missingness and stronger true correlations among outcomes, especially under the MAR missingness condition. These results matched those in Chang and Pituch's (2019) related, previous study. Chang and Pituch also found overestimated fixed-effect estimates, and this bias became more severe when the magnitude of the correlation and the degree of missingness increased. We generated datasets with positive correlations among outcomes (matching the pattern found in the real data analysis). We also introduced MAR missingness into the data such that smaller Outcome 1 scores led to a greater chance of missing Outcome 2 and Outcome 3 scores. Given scores among the three outcomes were positively correlated, it is more likely that lower Outcome 2 and Outcome 3 scores were then missing.

However, estimation of the multivariate model can handle missing at random (and completely at random) with both ML (e.g., Chang \& Pituch, 2019) and MCMC estimation (Browne, 2017). Although the multivariate models, MVHLM and MV-MMREM can handle missing data for multiple-outcomes simultaneously, the univariate model, Uni-MMREM, cannot handle the missing data as well because the analyses are conducted separately for each outcome and information about missing outcomes from related, complete outcome data is unavailable. Thus, it is not unexpected that the Uni-MMREM estimated means were higher on 
Table 4 Relative parameter bias $(R P B, \%)$ for Level 3 covariances by condition, ${ }^{\text {a }}$ estimated using the multivariate hierarchical linear models

\begin{tabular}{|c|c|c|c|c|c|c|c|}
\hline Correlation & ICC & School & Student & Mobile & $\sigma_{\mathrm{u} .12}$ & $\sigma_{\mathrm{u} .13}$ & $\sigma_{\mathrm{u} .23}$ \\
\hline \multirow[t]{16}{*}{$.3 / .5$} & \multirow[t]{8}{*}{.15} & \multirow[t]{4}{*}{50} & \multirow[t]{2}{*}{20} & .1 & -5.7 & -5.1 & -5.4 \\
\hline & & & & .4 & -30.2 & -28.6 & -30.2 \\
\hline & & & \multirow[t]{2}{*}{40} & .1 & -5.2 & -4.9 & -5.8 \\
\hline & & & & .4 & -28.9 & -29.8 & -28.9 \\
\hline & & \multirow[t]{4}{*}{100} & \multirow[t]{2}{*}{20} & .1 & -8.2 & -7.6 & -9.1 \\
\hline & & & & .4 & -32.7 & -31.6 & -32.5 \\
\hline & & & \multirow[t]{2}{*}{40} & .1 & -7.1 & -7.4 & -8.0 \\
\hline & & & & .4 & -32.2 & -31.3 & -32.7 \\
\hline & \multirow[t]{8}{*}{.25} & \multirow[t]{4}{*}{50} & \multirow[t]{2}{*}{20} & .1 & -2.5 & -1.8 & -7.4 \\
\hline & & & & .4 & -27.6 & -28.1 & -30.8 \\
\hline & & & \multirow[t]{2}{*}{40} & .1 & -5.00 & -4.7 & -7.8 \\
\hline & & & & .4 & -26.3 & -27.0 & -30.1 \\
\hline & & \multirow[t]{4}{*}{100} & \multirow[t]{2}{*}{20} & .1 & -5.5 & -5.9 & -9.1 \\
\hline & & & & .4 & -29.9 & -29.4 & -32.5 \\
\hline & & & \multirow[t]{2}{*}{40} & .1 & -6.1 & -5.6 & -10.2 \\
\hline & & & & .4 & -30.5 & -29.9 & -33.4 \\
\hline \multirow[t]{16}{*}{$.5 / .7$} & \multirow[t]{8}{*}{.15} & \multirow[t]{4}{*}{50} & \multirow[t]{2}{*}{20} & .1 & -3.9 & -4.9 & -5.3 \\
\hline & & & & .4 & -27.6 & -28.9 & -28.2 \\
\hline & & & \multirow[t]{2}{*}{40} & .1 & -7.3 & -7.4 & -6.7 \\
\hline & & & & .4 & -29.5 & -29.7 & -29.5 \\
\hline & & \multirow[t]{4}{*}{100} & \multirow[t]{2}{*}{20} & .1 & -8.6 & -9.1 & -8.6 \\
\hline & & & & .4 & -32.4 & -32.3 & -31.9 \\
\hline & & & \multirow[t]{2}{*}{40} & .1 & -8.6 & -8.9 & -8.1 \\
\hline & & & & .4 & -31.4 & -31.5 & -31.5 \\
\hline & \multirow[t]{8}{*}{.25} & \multirow[t]{4}{*}{50} & \multirow[t]{2}{*}{20} & .1 & -5.4 & -4.0 & -4.2 \\
\hline & & & & .4 & -29.4 & -28.3 & -28.7 \\
\hline & & & \multirow[t]{2}{*}{40} & .1 & -4.2 & -3.0 & -3.3 \\
\hline & & & & .4 & -29.8 & -28.9 & -28.8 \\
\hline & & \multirow[t]{4}{*}{100} & \multirow[t]{2}{*}{20} & .1 & -9.2 & -7.4 & -7.7 \\
\hline & & & & .4 & -31.3 & -30.5 & -30.4 \\
\hline & & & 40 & .1 & -8.9 & -7.9 & -7.3 \\
\hline & & & & .4 & -31.6 & -30.6 & $-\mathbf{3 0 . 7}$ \\
\hline
\end{tabular}

The $R P B$ values are bolded when the values are larger than $5 \%$ in magnitude. ${ }^{\text {a }}$ Results are summarized only for MAR missingness conditions because the other missingness consitions (MCAR or full dataset) had similar patterns. Results are presented only for the $40 \%$ missingness conditions; the pattern of results corresponded to the results with $20 \%$ missingness, although the bias was worse with 40 missingness.

Outcomes 2 and 3 (math and science in the real data) for the MAR datasets than for the complete data analyses (Chang \& Pituch, 2019).

Our results support the advantages of multivariate analyses with MAR data as suggested in previous, related studies that have reported that the use of multivariate models provides more power for fixed effects in scenarios with more missing data and for stronger correlations among outcomes increase. In real analyses, it is very likely that researchers will encounter some missing data and the data might well be missing at random. In such scenarios, applied researchers should not use multiple Uni-MMREMs because the fixed-effect parameter estimates will be biased. Instead, as encouraged by other methodological researchers (Chang \& Pituch, 2019; Park et al., 2015; Snijders \& Bosker, 2012) and supported by our results, analysts should use multivariate models paired with estimation procedures that can handle MAR data. Further methodological research is needed to evaluate the robustness of the MV-MMREM estimation under other patterns of missingness including missing not at random data.

In terms of the fixed-effect parameters' standard errors estimates, no substantial bias was found with either the UniMMREMs or the MV-MMREM. The standard error estimates were negatively biased under the MV-HLM for conditions with more mobile students. This result matches what Wolff Smith and Beretvas (2017) found when multiple-membership 
Table 5 Relative parameter bias $(R P B)$ for Level 3 variances by condition, ${ }^{a}$ estimated using multiple univariate multiple-membership random-effect models

\begin{tabular}{|c|c|c|c|c|c|}
\hline $\begin{array}{l}\% \\
\text { Missingness }\end{array}$ & Correlation & School & $\sigma_{u .11}^{2}$ & $\sigma_{u .22}^{2}$ & $\sigma_{u .33}^{2}$ \\
\hline \multirow[t]{4}{*}{$20 \%$} & \multirow[t]{2}{*}{$.3 / .5$} & 50 & 1.1 & -4.3 & -7.6 \\
\hline & & 100 & -0.3 & -6.1 & -8.7 \\
\hline & \multirow[t]{2}{*}{$.5 / .7$} & 50 & 1.4 & -13.4 & -16.7 \\
\hline & & 100 & 0.0 & -14.6 & -18.3 \\
\hline \multirow[t]{4}{*}{$40 \%$} & \multirow[t]{2}{*}{$.3 / .5$} & 50 & 1.5 & -4.6 & -7.8 \\
\hline & & 100 & -0.1 & -6.4 & -9.0 \\
\hline & \multirow[t]{2}{*}{$.5 / .7$} & 50 & 1.4 & -14.3 & -18.0 \\
\hline & & 100 & -0.2 & -15.5 & -19.3 \\
\hline
\end{tabular}

The $R P B$ values are bolded when the values are larger than $5 \%$ in magnitude. ${ }^{\text {a }}$ Results are summarized only for the missing-at-random (MAR) missingness conditions, because the other missingness conditions (missing completely at random or full dataset) had no substantial bias. The results are summarized across intraclass correlation, number of students, and mobility rate conditions, because these conditions did not substantially impact the results.

clusters were ignored in their comparison of a univariate MMREM with a univariate HLM. Also, even though in Meyers and Beretvas (2006), the authors compared a univariate cross-classified model to a univariate HLM that ignored cross-classified clusters, similar results (underestimation of standard errors for fixed effects) were also found. Misspecification of the multilevel model can result in misestimated standard errors. Researchers note that ignoring a level in a dataset's structure can influence not only the variance component estimates but also the fixed effects' $S E$ estimates (e.g., Luo \& Kwok, 2009; Raudenbush \& Bryk, 2002; Van den Noortgate, Opdenakker, \& Onghena, 2005). Not surprisingly, then, the implications of ignoring the multiplemembership data structure will impact associated statistical inferences. Underestimated standard errors will lead to inflated Type I error rates. Thus, as with univariate analyses, when using multivariate model analyses, multiple-membership data structures should not be ignored due to the potential impact on standard error estimates and resulting statistical inferences.

The Uni-MMREMs estimates of Level 2 and Level 3 variance components were negatively biased for MAR data and only for the outcomes with missing data (Outcomes 2 and 3). This bias was larger in conditions in which the true correlations among outcomes was larger. These results matched those found in Chang and Pituch (2019). These results make sense because of the pattern of the missing data that was introduced. Lower scores were those most likely to be missing and the missing data deflates the variability of the resulting scores as compared to the true value before missing data were introduced.

Under MV-HLM estimation, Level 3 variance and covariance parameter estimates were substantially negatively biased

Table 6 Summary of simulation study results by conditions and estimating models

\begin{tabular}{|c|c|c|c|}
\hline Estimates & MV-HLM & Univariate MMREM & MV-MMREM \\
\hline Fixed effects & No bias & $\begin{array}{l}\text { Positive bias } \\
\text { - MAR data } \\
\text { - Higher proportion of missingness } \\
\text { - Stronger correlation among outcomes }\end{array}$ & No bias \\
\hline$S E$ of fixed effects & $\begin{array}{l}\text { Negative bias } \\
\text { - Larger mobile rate }\end{array}$ & No bias & No bias \\
\hline Level 2 variance & No bias & $\begin{array}{l}\text { Negative bias } \\
\text { - MAR data } \\
\text { - Stronger correlation among outcomes }\end{array}$ & No bias \\
\hline Level 2 covariance & No bias & N/A & No bias \\
\hline Level 3 variance & $\begin{array}{l}\text { Negative bias } \\
\text { - All conditions }\end{array}$ & $\begin{array}{l}\text { Negative bias } \\
\text { - MAR data } \\
\text { - Stronger correlation among outcomes }\end{array}$ & No bias \\
\hline Level 3 covariance & $\begin{array}{l}\text { Negative bias } \\
\text { - All conditions }\end{array}$ & N/A & $\begin{array}{l}\text { Positive bias } \\
\text { - Smaller number of schools }\end{array}$ \\
\hline
\end{tabular}

MV-HLM, multivariate hierarchical linear model; MMREM, multiple-membership random-effect model; MV-MMREM, multivariate multiplemembership random-effect model. 
across all conditions and especially in conditions with more mobility. Previous studies (Chung \& Beretvas, 2012; Wolff Smith \& Beretvas, 2017) that conducted simulations studies to examine the impact of ignoring multiple-membership data structures in multilevel models also found underestimation of school-level variance components with higher mobility. As we explained in Chung and Beretvas, the school-level residuals are assumed normally distributed with variance $\sigma_{u}^{2}$. In multiple-membership data, here, the overall Level 2 residual for a mobile individual is a function of multiple Level 2 units. The variance of the resulting weighted sum of residuals (where the weights sum to one and are thus each less than one) for mobile individuals will be less than the variance, $\sigma_{u}^{2}$, for nonmobile individuals. The MMREM models (Uni-MMREM and MV-MMREM) are designed specifically to handle this difference and thus the resulting Level 2 residuals' variance is adjusted accordingly when these multiple-membership models are estimated. This is not the case with the conventional multilevel model. The observed variance of the residuals for mobile individuals will still be smaller than for nonmobile individuals and the resulting single variance, $\sigma_{u}^{2}$, estimated using a conventional multilevel model (here, the MVHLM) will then be smaller than it should be (see Chung \& Beretvas, 2012).

Level 2 (student-level) covariance components under the MV-HLM were overestimated when there were a greater proportion of mobile students and larger true ICCs. The relative parameter bias in Level 2 variances component estimates tended to be positive even though the degree of bias was not substantial. Several studies have examined the consequences of ignoring a level of nesting in multilevel data (Moerbeek, 2004; Tranmer, 2001; Van den Noortgate et al., 2005; Van Landeghem, De Fraine, \& Van Damme, 2005), and results have shown that when clustering at the highest level of the data structure is ignored, the associated variance component is added (redistributed) to the variance component at the adjunct, lower level. Although these previous studies examined the results of ignoring the highest level (here, the school level) of clustering entirely, our model (MV-HLM) did not completely ignore the highest level, but only recognized one of the set of multiple clusters (schools) associated with the participants (students). The resulting impact is the same with the ignored cluster effects being pushed to the lower level (here, the student level) resulting in overestimation of student-level variance-covariance estimates.

Previous studies (Chung \& Beretvas, 2012; Meyers \& Beretvas, 2006) that focused on misspecification of upper level clusters (e.g., multiple membership clusters and cross-classified clusters) also found overestimation of participant level (Level 2) variance components. Note, however, that these previous researchers only examined univariate models and thus considered estimation only of variance not covariance components. Despite this, overestimation of Level 2 residuals' variance components also seem likely to co-occur with substantial overestimation of Level 2 covariance components as was found in the present study.

Interestingly, the MV-MMREM estimates of Level 3 covariance components were positively biased in conditions with fewer schools. These results match those from previous studies (Chung \& Beretvas; 2012; Leckie, 2009; Wolff Smith $\&$ Beretvas, 2017) that examined univariate (not multivariate) models. These previous studies also found overestimation in cluster-level variances even under the correctly specified MMREM. This research also found that when the multiplemembership data structure was ignored, the resulting clusterlevel variances were more underestimated in conditions with more clusters. According to Browne and Draper (2000) evaluating MCMC estimation, variance component estimates tend to be overestimated in conditions with fewer clusters (matching what we found with the MV-MMREM estimates). In addition, MV-HLM estimation of Level 3 variancecovariance components were more negatively biased in conditions with more Level 3 units (larger number of schools). The smaller degree of negative bias found with fewer rather than more clusters under the MV-HLM could have occurred because of MCMC's tendency to overestimate cluster-level variances with fewer clusters being counteracted by the negative bias that can result from model misspecification (Chung \& Beretvas, 2012; Wolff Smith \& Beretvas, 2017).

Given these results, use of the MV-HLM is not recommended if the data have a multiple-membership data structure because the standard errors for fixed effects as well as the Level 3 variances and covariances are negatively biased across conditions. In addition, with datasets consisting of multiple-related outcomes, estimating a set of UniMMREMs works well only if the outcomes are not related or the dataset does not have missing data. Because most applied researchers are primarily concerned with fixed-effect estimates, use of Uni-MMREMs is not recommended if there are missing outcomes and if the researcher cannot be sure whether the missingness is based on the unlikely MCAR assumption with MAR or MNAR missingness being more likely. On the other hand, the MV-MMREM performs well across all of conditions examined here except in scenarios with too few schools (clustering units) with slight overestimation found for Level 3 covariance estimates. Because the present study found some over-estimation with 50 clustering units and no bias with 100 clusters, future research should examine conditions with different numbers of clustering units to help refine the recommendation for the minimum number of clustering units.

Some limitations of the present study need to be considered. The results of this study should not be generalized beyond the limited conditions examined. Different numbers of clusters and students per cluster could impact results as well as different patterns of ICC and correlation values. Also, only 
one type of prior distributions for MCMC estimation was used; however, future research could examine use of different prior distributions. In addition, only a very simple baseline MV-MMREM model was used to generate the data. In addition, although DIC values seem to work well for choosing between MV-MMREM and non-MMREM models, the performance of DIC values for choosing among multiple MVMMREM has not been tested. Future research can be designed to take on each of these limitations. Finally, in the present study we only considered MCMC estimation but other estimation methods (e.g., likelihood-estimation) with other statistical programs, such as xxM (Mehta, 2013) or lme4 (Bates, Maechler, Bolker, \& Walker, 2015), should be examined. On the basis of the present study, future research can extend these more complicated models to examine the handling of multivariate data in scenarios with multiplemembership data.

Even though the data generated in the present simulation study sound quite complex in their structure (multivariate, with multiple memberships and MAR missingness), these kinds of data seem very likely to be encountered in real educational and social science research. Therefore, the findings of the present study supporting the use of MV-MMREM over both MV-HLM and multiple Uni-MMREMs should prove helpful to applied researchers who encounter these kinds of methodological dilemmas.

\section{References}

Baldwin, S. A., Imel, Z. E., Braithwaite, S. R., \& Atkins, D. C. (2014). Analyzing multiple outcomes in clinical research using multivariate multilevel models. Journal of Consulting and Clinical Psychology, $82,920-930$.

Bassiri, D. (1988). Large and small sample properties of maximum likelihood estimates for the hierarchical linear model (Doctoral dissertation).

Bates, D., Maechler, M., Bolker, B., \& Walker, S. (2015). lme4: Linear mixed-effects models using Eigen and S4 (R package version). Retrieved from http://cran.r-project.org/package=lme4

Beretvas, S. N. (2015). Uncovering processes and pathways in familyschool research: Modeling innovations for handling data complexities. In S. M. Sheridan \& E. M. Kim (Eds.), Processes and pathways of family-school partnerships across development (pp. 81-99). Berlin, Germany: Springer.

Beretvas, S. N., \& Murphy, D. L. (2013). An evaluation of information criteria use for correct cross-classified random effects model selection. Journal of Experimental Education, 81, 429-463.

Browne, W. J. (2017). MCMC Estimation in MLwiN v3.00. Bristol, UK: University of Bristol, Centre for Multilevel Modelling.

Browne, W. J., \& Draper, D. (2000). Implementation and performance issues in the Bayesian and likelihood fitting of multilevel models. Computational Statistics, 15, 391-420.

Chandola, T., Clarke, P., Wiggins, R. D., \& Bartley, M. (2005). Who you live with and where you live: Setting the context for health using multiple-membership multilevel models. Journal of Epidemiology and Community Health, 59, 170-175.
Chang, W. (2015). Sufficient sample sizes for the multivariate multilevel regression model (Doctoral dissertation). Austin, TX: University of Texas. http://hdl.handle.net/2152/31009

Chang, W., \& Pituch, K. A. (2019). The performance of multilevel models when outcome data are incomplete. Journal of Experimental Education, 87, 1-16. doi:https://doi.org/10.1080/ 00220973.2017 .1377676

Chung, H., \& Beretvas, S. N. (2012). The impact of ignoring multiplemembership data structures in multilevel models. British Journal of Mathematical and Statistical Psychology, 65, 185-200.

Clarke, P., \& Wheaton, B. (2007). Addressing data sparseness in contextual population research. Sociological Methods and Research, 35, 311-351.

Cools, W., Van den Noortgate, W., \& Onghena, P. (2009). Design efficiency for imbalanced multilevel data. Behavior Research Methods, 41, 192-203. doi:https://doi.org/10.3758/BRM.41.1.192

Enders, C. K. (2001). The performance of the full information maximum likelihood estimator in multiple regression models with missing data. Educational and Psychological Measurement, 61, 713-740.

Gelman, A., \& Rubin, D. B. (1992). Inference from iterative simulation using multiple sequences. Statistical Science, 7, 457-472. doi: https://doi.org/10.2307/2246093

Goldstein, H. (2010). Multilevel statistical models (4th ed.). New York, NY: Hodder Arnold.

Goldstein, H., Burgess, S., \& McConnell, B. (2007). Modelling the effect of pupil mobility on school differences in educational achievement. Journal of the Royal Statistical Society: Series A, 170, 941-954.

Gruman, D. H., Harachi, T. W., Abbott, R. D., Catalano, R. F., \& Fleming, C. B. (2008). Longitudinal effects of student mobility on three dimensions of elementary school engagement. Child Development, 79, 1833-1852.

Hedges, L. V., \& Hedberg, E. C. (2007). Intraclass correlation values for planning group-randomized trials in education. Educational Evaluation and Policy Analysis, 29, 60-87.

Hoogland, J. J., \& Boomsma, A. (1998). Robustness studies in covariance structure modeling. Sociological Methods and Research, 26, $329-367$.

Hox, J. (2010). Multilevel analysis: Techniques and applications (2nd ed.). Hoboken, NJ: Taylor \& Francis.

Hox, J., \& Maas, C. J. (2001). The accuracy of multilevel structural equation modeling with pseudobalanced groups and small samples. Structural Equation Modeling, 8, 157-174.

Kerbow, D. (1996). Patterns of urban student mobility and local school reform. Journal of Education for Students Placed at Risk, 1, 147169.

Kim, K.-S. (1990). Multilevel data analysis: A comparative examination of analytical alternatives (Doctoral dissertation). Available from ProQuest Dissertations and Theses database. (UMI No. 9035243)

Konstantopoulos, S. (2010). Power analysis in two-level unbalanced designs. Journal of Experimental Education, 78, 291-317.

Leckie, G. (2009). The complexity of school and neighborhood effects and movements of pupils on school differences in models of educational achievement. Journal of the Royal Statistical Society: Series A, 172, 537-554.

Luo, W., \& Kwok, O. (2009). The impacts of ignoring a crossed factor in analyzing cross-classified data. Multivariate Behavioral Research, 44, 182-212.

Maas, C. J. M., \& Hox, J. J. (2004). Robustness issues in multilevel regression analysis. Statistica Neerlandica, 58, 127-137.

Maas, C. J. M., \& Hox, J. J. (2005). Sufficient sample sizes for multilevel modeling. Methodology, 1, 86-92. doi:https://doi.org/10.1027/ 1614-2241.1.3.86

Mathieu, J. E., Aguinis, H., Culpepper, S. A., \& Chen, G. (2012). Understanding and estimating the power to detect cross-level interaction effects in multilevel modeling. Journal of Applied Psychology, 97, 951-966. 
McNeish, D., \& Stapleton, L. (2013, April). The effect of the number of clusters on multilevel model estimates: A review and illustration. Paper presented at the meeting of the American Educational Research Association, San Francisco, CA.

Mehta, P. D. (2013). xxM user's guide. Houston, TX: University of Houston.

Meyers, J. L., \& Beretvas, S. N. (2006). The impact of inappropriate modeling of cross-classified data structures. Multivariate Behavioral Research, 41, 473-497.

Moerbeek, M. (2004). The consequence of ignoring a level of nesting in multilevel analysis. Multivariate Behavioral Research, 39, 129149.

Mok, M. (1995). Sample size requirements for 2-level designs in educational research. Multilevel Modelling Newsletter, 7, 11-15.

Park, R., Pituch, K. A., Kim, J., Chung, H., \& Dodd, B. G. (2015). Comparing the performance of multivariate multilevel modeling to traditional analyses with complete and incomplete data. Methodology, 11, 100-109.

Pituch, K. A., Whittaker, T. A., \& Chang, W. (2016). Multivariate models for normal and binary responses in intervention studies. American Journal of Evaluation, 37, 270-286.

Pollack, J. M., Atkins-Burnett, S., Najarian, M., \& Rock, D. A. (2005). Early Childhood Longitudinal Study, kindergarten class of 1998-99 (ECLS-K), psychometric report for the fifth grade (NCES 2006036). Washington, DC: National Center for Education Statistics.

Rasbash, J., \& Browne, W. J. (2001). Modeling non-hierarchical structures. In A. H. Leyland \& H. Goldstein (Eds.), Multilevel modelling of health statistics (pp. 93-105). Chichester, UK: Wiley.

Raudenbush, S. W., \& Bryk, A. S. (2002). Hierarchical linear models: Applications and data analysis methods (2nd ed.). Thousand Oaks, CA: Sage.

Raudenbush, S. W., \& Liu, X. (2000). Statistical power and optimal design for multisite randomized trials. Psychological Methods, 5, 199-213.

R Development Core Team. (2010). R: A language and environment for statistical computing. Vienna, Austria: R Foundation for Statistical Computing. Retrieved from www.R-project.org

Reise, S. P., \& Duan, N. (2003). Multilevel modeling: Methodological advances, issues, and applications. Hove, UK: Psychology Press.
Scherbaum, C. A., \& Ferreter, J. M. (2009). Estimating statistical power and required sample sizes for organizational research using multilevel modeling. Organizational Research Methods, 12, 347-367.

Shi, Y., Leite, W. L., \& Algina, J. (2010). The impact of omitting the interaction between crossed factors in cross-classified random effects modelling. British Journal of Mathematical and Statistical Psychology, 63, 1-15.

Snijders, T. A., \& Bosker, R. J. (2012). Multilevel analysis: An introduction to basic and advanced multilevel modeling. Thousand Oaks, CA: Sage.

Spiegelhalter, D. J., Best, N. G., Carlin, B. P., \& Van der Linde, A. (2002). Bayesian measures of model complexity and fit. Journal of the Royal Statistical Society: Series B, 64, 583-639.

Tabachnick, B. G., Fidell, L. S., \& Osterlind, S. J. (2001). Using multivariate statistics (4th ed.). Needham Heights: Allyn \& Bacon.

Tranmer, M. (2001). Ignoring a level in a multilevel model: Evidence from UK census data. Environment and Planning, 33, 941-948.

Whittaker, T. A., \& Furlow, C. F. (2009). The comparison of model selection criteria when selecting among competing hierarchical linear models. Journal of Modern Applied Statistical Methods, 8, 173 193.

Wolff Smith, L. J., \& Beretvas, S. N. (2014). The impact of using incorrect weights with the multiple-membership random effects model. Methodology, 10, 31-42.

Wolff Smith, L. J., \& Beretvas, S. N. (2017). A comparison of techniques for handling and assessing the influence of mobility on student achievement. Journal of Experimental Education, 85, 3-23.

Van den Noortgate, W., Opdenakker, M. C., \& Onghena, P. (2005). The effects of ignoring a level in multilevel analysis. School Effectiveness and School Improvement, 16, 281-303.

Van Landeghem, G., De Fraine, B., \& Van Damme, J. (2005). The consequence of ignoring a level of nesting in multilevel analysis: A comment. Multivariate Behavioral Research, 40, 423-434.

Zhang, Z., Parker, R., Charlton, C. M., Leckie, G., \& Browne, W. J. (2016). R2MLwiN: A package to run MLwiN from within R. Journal of Statistical Software, 72(10), 1-43.

Publisher's note Springer Nature remains neutral with regard to jurisdictional claims in published maps and institutional affiliations. 